\title{
Simulación y experimentación de laminación plana de placas de aluminio 6063
}

\author{
T. Robert*, O. Vázquez y E. López \\ Departamento Ingeniería Metalúrgica, Facultad de Química, Universidad Nacional Autónoma de México, \\ Cto. Institutos Ed. D, Fac. Quim., Cd. Universitaria, México D.F. 04510, México. \\ E-mail: robertn@unam.mx
}

(recibido/received: 31-Enero-2011; aceptado/accepted: 10-Junio-2011)

\begin{abstract}
RESUMEN
Se generó una metodología experimental para el entendimiento teórico-práctico de los conceptos del proceso de laminación plana en frío. Se laminaron placas de aluminio de aproximadamente $100 \mathrm{~mm}$ de longitud y $9 \mathrm{~mm}$ de espesor, a diferentes anchos: 10 y $30 \mathrm{~mm}$. Se seleccionó una razón de reducción constante de $0.3 \mathrm{~mm}$ para cada paso de laminación, hasta lograr una reducción superior al 80 \% en espesor. Se implementó un modelo matemático en el paquete comercial Abaqus ${ }^{\circledR}$ con el fin de obtener un mayor entendimiento sobre la influencia de las variables del proceso (fuerza aplicada y dimensiones de las probetas) en los campos de esfuerzos y deformaciones que, experimentalmente, no se pueden observar. Se encontró, mediante la simulación matemática, que se produce un endurecimiento superficial en las placas y que la deformación obtenida es heterogénea, lo cual se corrobora físicamente.
\end{abstract}

Palabras claves: Laminación plana; Aluminio 6063-Al; Sistema de adquisición de datos; Simulación

\begin{abstract}
An experimental methodology was generated for understanding the theoretical and practical concepts inherent to cold flat rolling. Aluminium slabs about $100 \mathrm{~mm}$ length, $9 \mathrm{~mm}$ thickness with different widths: 10 and $30 \mathrm{~mm}$ were rolled. A constant $0.3 \mathrm{~mm}$ thickness reduction step was performed in order to achieve a thickness reduction greater than $80 \%$. In the Abaqus ${ }^{\circledR}$ commercial software, a mathematical model was implemented in order to obtain a greater understanding concerning how the process variables affect the fields of stresses and strains that are not observed experimentally. It was found, by mathematical simulation, that a superficial hardening in the plates was produced and the deformation obtained is heterogeneous, which is corroborated physically.
\end{abstract}

Keywords: Flat rolling; 6063 Aluminium; Data acquisition system; Simulation

*Autor para la correspondencia 


\section{INTRODUCCIÓN}

El proceso de laminación plana en frío es uno de los procesos de conformado mecánico más estudiado desde el siglo pasado, aunque su origen data desde el siglo XV (Swank, 1892). No obstante, sigue siendo un proceso vigente $\mathrm{y}$ en constante evolución. El aprendizaje del proceso involucra un interés principal debido a su complejidad a nivel industrial.

El proceso de laminación plana en frío es un proceso de compresión directa, donde la fuerza de compresión se aplica sobre la superficie de una pieza plana permitiendo que el metal fluya en ángulos rectos respecto a la dirección de compresión (Dieter, 1986).

Con el fin de relacionar los aspectos teóricos y prácticos del proceso se desarrolló una metodología de medición capaz de determinar cuantitativamente la distribución de presión ejercida por el rodillo hacia el material a laminar (Lozada, 2009). Para ésto se instrumentó una laminadora semi-industrial OAM M120 de 1 HP, utilizando una celda de carga y un adquisidor de datos para registrar in situ la diferencia de potencial producida por el cambio dimensional del material durante la reducción de espesor.

Durante el proceso de laminación plana se pueden analizar los cambios de espesor de las placas en función del número de pasos y el cambio de la fuerza necesaria para producir deformación permanente, pero no se puede analizar in situ si la deformación producida es homogénea o heterogénea, es decir, no se puede observar la evolución de los campos de esfuerzos y de deformaciones desarrollados. Para realizar esto se necesita de técnicas experimentales auxiliares para determinar los campos de esfuerzos y deformaciones residuales. Ejemplo de éstas técnicas son: difracción de rayos $x$, hole drilling, y más recientemente nanoindentación (Khan et al., 2010). El empleo de estas pruebas tiene un coste elevado debido a la disponibilidad del equipo especializado, además de que la preparación de las muestras no es trivial. Una alternativa para el estudio de los estados de esfuerzos y deformaciones es la modelación matemática por medio de elementos finitos (Duan y Sheppard, 2002; Riahifar y Serajzadeh, 2007).

\section{Laminación plana}

Para el control del proceso es necesario establecer las relaciones geométricas y de fuerza involucradas durante la deformación de un metal mediante el proceso de laminación plana en frío, Fig. 1. Una placa de metal con espesor inicial $h_{0}$ es introducida a un molino de laminación, con una velocidad inicial $v_{0}$; la cual pasa a través de los rodillos y sale con un espesor reducido $h_{f}$. En la condición de deformación plana, el ancho de la placa no se incrementa con la deformación producida por el rodillo, por lo que la compresión vertical producida es traducida completamente en una elongación en la dirección longitudinal o de laminación. El volumen se conserva a lo largo del paso de laminación por unidad de tiempo y el balance de fuerzas establece que:

$$
b h_{0} v_{0}=b h v=b h_{f} v_{f}
$$

donde $b$ es el ancho de la placa, $v_{f}$ es la velocidad final o de salida de la placa.

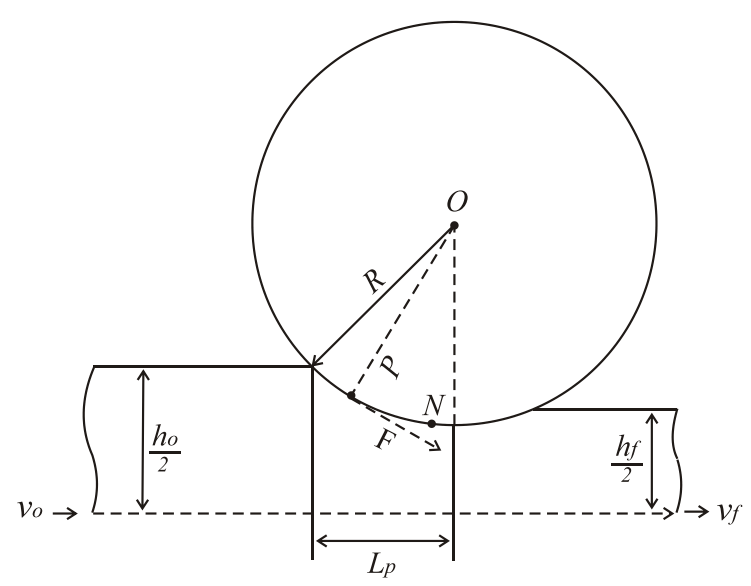

Fig. 1 Representación geométrica y de las fuerzas que actúan en el proceso de laminación plana.

Dado que se considera que el ancho de la placa no se deforma, se requiere que la velocidad de salida, $v_{f}$, sea mayor que la velocidad de entrada, $v_{0}$. La velocidad de la placa debe incrementarse 
paulatinamente desde la entrada hasta la salida, Fig. 1. Sólo hay un punto a lo largo de la superficie de contacto entre el rodillo y la placa, en el cual las velocidades son iguales y se conoce como punto neutro $N$. En general, en este punto se obtiene la mayor aplicación de fuerza.

En cualquier punto sobre la superficie de contacto actúan dos fuerzas sobre la placa de metal: la fuerza radial $P_{r}$ y la fuerza de fricción tangencial $F$, Fig. 1. La fuerza de fricción tangencial actúa en la dirección de laminación antes del punto neutro donde la velocidad de la placa es menor a la velocidad del rodillo; después de este punto la fuerza de fricción actúa en dirección contraria debido a que la velocidad de la placa es superior a $v_{r}$. Por otro lado, la componente vertical $P_{r}$ es conocida como la fuerza de laminación $P$. La fuerza de laminación es la fuerza ejercida por el rodillo hacia la placa. La presión específica del rodillo es la fuerza de laminación aplicada, dividida entre el área de contacto y, a su vez, el área de contacto $A_{c}$ es igual al producto del ancho de la placa $b$ por la longitud proyectada del arco de contacto $L_{p}$,

$L_{p} \approx\left[R\left(h_{0}-h_{f}\right)\right]^{\frac{1}{2}}$

Por lo tanto, la presión específica del rodillo está dada por:

$$
p=\frac{P}{b L_{p}}
$$

Debido a que la fuerza de laminación varía a lo largo de la longitud de contacto por la fuerza de fricción tangencial y la velocidad de la placa, es necesario determinar un valor medio. Mediante la implementación de un sistema de adquisición de datos, en $\mathrm{mV}$, es posible medir experimentalmente la diferencia de potencial relacionada con la fuerza de laminación en función del tiempo y obtener así un valor promedio de la fuerza. Para esto se utiliza el teorema del valor medio,

$$
P_{m}=\frac{\int P(t) d t}{\int d t}
$$

Por consiguiente, la presión media específica del rodillo se calcula como:

$$
p_{m}=\frac{P_{m}}{b L_{p}}
$$

Para determinar las deformaciones producidas en la placa, en cada paso de laminación, se utiliza la expresión de la deformación real:

$$
\begin{aligned}
\varepsilon_{h} & =\ln \left(\frac{h}{h_{0}}\right) \\
\varepsilon_{l} & =\ln \left(\frac{l}{l_{0}}\right) \\
\varepsilon_{h} & =\ln \left(\frac{b}{b_{0}}\right)
\end{aligned}
$$

donde $h, l$ y $b$ son las dimensiones instantáneas en espesor, largo y ancho respectivamente, después de cada paso de laminación.

\section{METODOLOGÍA}

Se maquinaron placas de aluminio 6063 de aproximadamente $100 \mathrm{~mm}$ de largo y $9 \mathrm{~mm}$ de espesor, a diferentes anchos: 10 y $30 \mathrm{~mm}$. La composición química del aluminio 6063 utilizado se muestra en la Tabla 1.

Tabla 1 Composición de la aleación 6063-Al.

\begin{tabular}{ccccc}
\hline 6063-Al & $\mathrm{Si}$ & $\mathrm{Mg}$ & $\mathrm{Fe}$ & Otros \\
\hline \% peso & 0.346 & 0.438 & 0.213 & 0.0345 \\
\hline
\end{tabular}

Para la deformación de las placas se utilizó una laminadora semi-industrial OAM M120, de 1 HP. La velocidad del rodillo se mantuvo constante en $18.5 \mathrm{rpm}$ con un radio de $32.5 \mathrm{~mm}$. La razón de reducción se fijo en $0.3 \mathrm{~mm}$ para cada paso de laminación hasta lograr una reducción en espesor ligeramente superior al 80 $\%$ de trabajado en frío.

El valor de la fuerza ejercida por el rodillo, en cada paso de laminación, fue obtenida por una celda de carga Transducer Techniques LBO$10 \mathrm{~K}$, de $45 \mathrm{kN}$ y una tarjeta de adquisición de datos IOTECH PD/55. 
Para obtener una curva de calibración que permitiera la correlación entre el voltaje generado y la fuerza aplicada fue necesario realizar un ensayo de compresión en una maquina universal de ensayos mecánicos marca MTS, modelo 810, con el sistema de adquisición de datos Testlink versión 2.0. La Fig. 2 muestra los resultados obtenidos de la curva de calibración de la celda de carga.

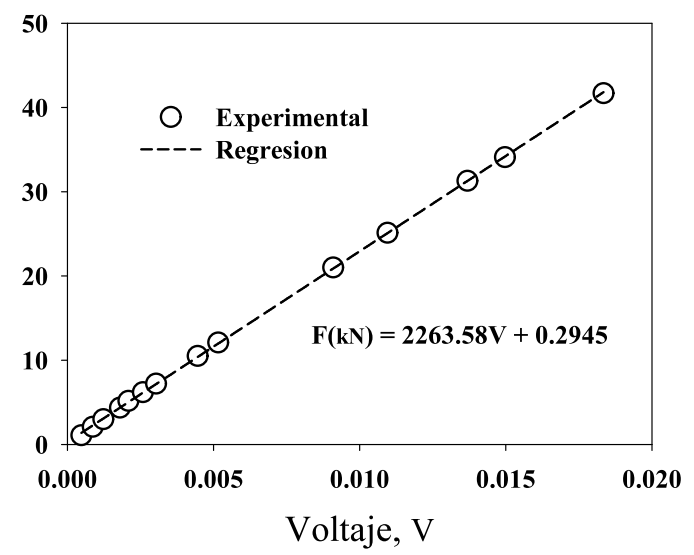

Fig. 2 Curva de calibración de la celda de carga.

El sistema de adquisición de datos permite obtener una curva de distribución de voltaje con respecto al tiempo, la cual se correlaciona mediante la curva de calibración, Fig. 2, para encontrar una función del tipo $P(t)$ y aplicar el teorema del valor medio para, finalmente, construir una curva de distribución de fuerza a lo largo de la longitud de contacto y, así, determinar el valor promedio de la fuerza, $P_{m}$, Fig. 3.

La Fig. 3 muestra las curvas de distribución de fuerza durante un paso de laminación para las placas de 10 y $30 \mathrm{~mm}$ de ancho. Se observa que a lo largo de la longitud de contacto se mantiene casi constante la distribución de la fuerza y que se incrementa proporcionalmente al ancho de la placa. Las presiones medias específicas del rodillo, al igual que la fuerza, se incrementan en proporción al ancho de la placa. El área bajo la curva representa la energía requerida para deformar al material y está asociada con el valor del torque y potencia requerida. La potencia desarrollada en el proceso de laminación está distribuida en: a) energía requerida para deformar el material, b) energía necesaria para vencer las fuerzas de fricción entre la pieza de trabajo y la superficie de los rodillos, c) perdida de energía en los piñones y el sistema de transmisión y d) las pérdidas eléctricas en el motor (Dieter, 1986).

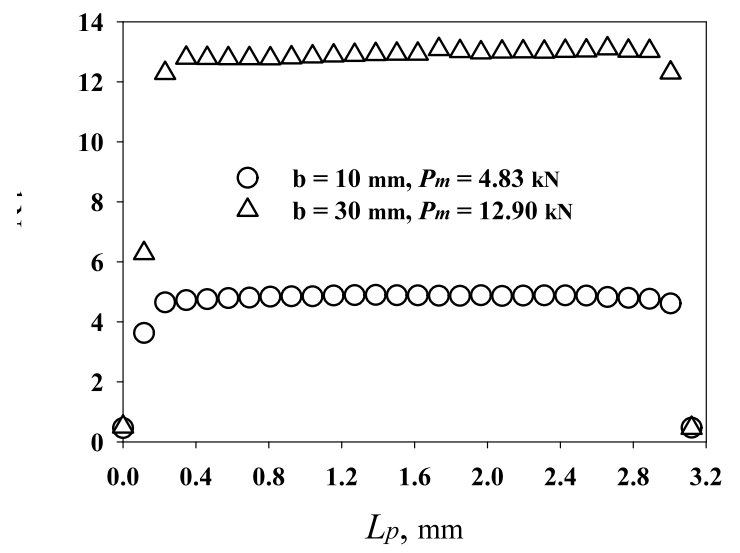

Fig. 3 Curvas de distribución de fuerza a lo largo de la longitud de contacto a diferentes anchos de placa, 10 y $30 \mathrm{~mm}$.

La Tabla 2 muestra los valores calculados para el torque y la potencia desarrollada.

Tabla 2 Parámetros de operación del proceso de laminación en frío.

\begin{tabular}{ccccc}
\hline $\begin{array}{c}\text { Torque, } \\
k N m\end{array}$ & $\begin{array}{c}\text { Potencia, } \\
h p\end{array}$ & $\begin{array}{c}b, \\
m m\end{array}$ & $\begin{array}{c}\text { Reducción } \\
\text { espeso, } r \\
\Delta h, m m\end{array}$ & $\begin{array}{c}V_{r} \\
\text { rpm }\end{array}$ \\
\hline 13.57 & 0.035 & 10 & 0.3 & 18.5 \\
\hline 36.22 & 0.094 & 30 & 0.3 & 18.5 \\
\hline
\end{tabular}

La solución numérica del proceso de laminación requiere de la determinación del coeficiente de fricción y de las propiedades mecánicas del material. Estas propiedades, en especial la resistencia a la fluencia, necesitan ser determinadas experimentalmente como una función de la rapidez de deformación; por lo que se maquinaron probetas de compresión para determinar las propiedades mecánicas. La Tabla 3 presenta las dimensiones iniciales de las probetas y las condiciones de prueba.

Los resultados de los ensayos de compresión se presentan en la Figura 4. 
Tabla 3 Dimensiones iniciales de las probetas de compresión y velocidad de deformación ensayada a cada probeta.

\begin{tabular}{cccc}
\hline $\begin{array}{c}\text { No. } \\
\text { Probeta }\end{array}$ & $\begin{array}{c}\text { Diámetro } \\
\text { inicial, } \\
\mathrm{mm}\end{array}$ & $\begin{array}{c}\text { Altura } \\
\text { inicial, } \\
\mathrm{mm}\end{array}$ & $\begin{array}{c}\text { Rapidez } \\
\text { def. real } \\
\text { promedio, } \\
\mathrm{s}^{-1}\end{array}$ \\
\hline 1 & 8.18 & 6.46 & 0.041 \\
\hline 2 & 8.15 & 6.48 & 0.460 \\
\hline
\end{tabular}

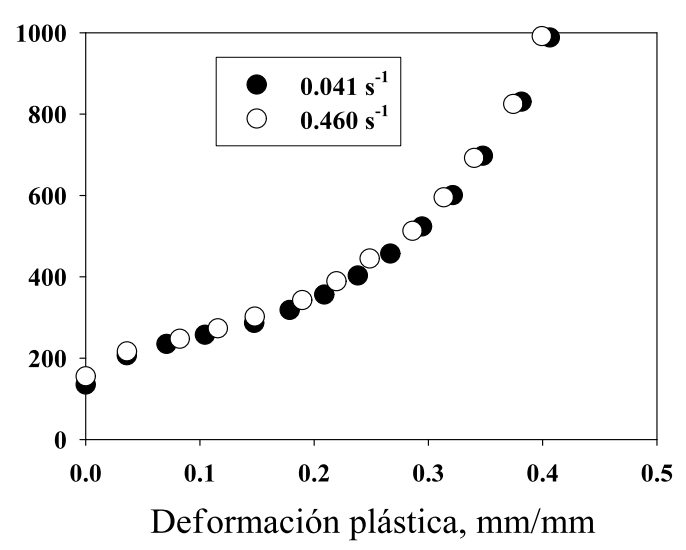

Fig. 4 Curvas esfuerzo-deformación de las probetas de compresión en función de la rapidez de deformación.

Se podría pensar que el material es poco sensible a la rapidez de deformación pero, analizando los datos, se observa que para una rapidez de deformación de $0.041 \mathrm{~s}^{-1}$ se obtiene una resistencia a la fluencia de $135 \mathrm{MPa}$, mientras que para una rapidez de deformación de $0.46 \mathrm{~s}^{-1}$ se obtiene una resistencia a la fluencia de 155 $\mathrm{MPa}$. Por lo que el material presenta un comportamiento esperado, es decir, las propiedades y comportamiento mecánico son función de la rapidez de deformación.

\section{Simulación computacional}

Para obtener un mejor entendimiento del proceso de laminación, con respecto a los campos de esfuerzos y deformaciones, se implementó un modelo matemático usando el paquete comercial Abaqus ${ }^{\circledR}$, el cual se basa en el método de elemento finito.
La simulación por medio de elementos finitos es una herramienta utilizada para analizar procesos de conformado mecánico. Una vez que se genera un modelo, las variables independientes del proceso pueden ser modificadas para estudiar su efecto sobre las variables dependientes. Se consideran dos aspectos en la simulación: suposiciones del sistema de deformación y formulación matemática.

a) Suposiciones del sistema de deformación

- Problema en 2D, es decir, prevalecen las condiciones de deformación plana.

- Existe simetría con respecto al eje longitudinal, por lo que sólo la mitad del sistema se modela.

- Comportamiento elasto - plástico e isotrópico de la placa.

- Comportamiento rígido del rodillo.

- Durante la deformación plástica no existen cambios de volumen en la placa.

- La respuesta mecánica en la zona elástica sigue la ley de Hooke.

- Las propiedades mecánicas dependen de la rapidez de deformación.

- Se considera la fuerza de fricción entre el rodillo y la placa.

- No se considera ningún tipo de fuerza de cuerpo.

- No se genera calor por deformación ni por fricción.

b) Formulación matemática

Las ecuaciones de equilibrio en forma integral para un elemento de volumen $V$ y superficie $S$ y considerando la fórmula de Cauchy para esfuerzos (Chen y Han, 1988) son:

$\int_{S} n_{i} \sigma_{i j} d S+\int_{V} F_{i} d V=0$

donde $\sigma_{i j}$ es el tensor de esfuerzos y $F_{i}$ es cualquier fuerza de cuerpo. Sin considerar fuerzas de cuerpo, el sistema de ecuaciones diferenciales de equilibrio se obtiene aplicando el teorema de la divergencia y resolviendo en un volumen arbitrario: 


$$
\sigma_{i j, j}=0
$$

Para obtener el campo de deformaciones se resuelve el campo de desplazamiento y, con ecuaciones constitutivas, se obtiene el campo de esfuerzos.

La Ecuación (9) establece el incremento de esfuerzo para un determinado incremento de deformación,

$$
d \sigma_{i j}=C_{i j k l}^{e p} d \varepsilon_{k l}
$$

donde $\varepsilon_{k l}$ es el tensor de rigidez elástico-plástico, que es función del estado de esfuerzos y de la historia de carga aplicada. La Fig. 5 muestra las condiciones a la frontera necesarias para la solución del problema.

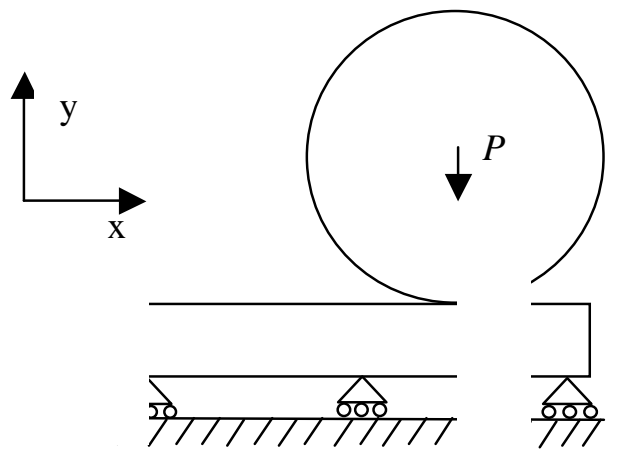

Fig. 5 Condiciones a la frontera del proceso de laminación. Nota: el diagrama no está a escala.

Para el modelo de simulación se utilizaron los datos de las curvas esfuerzo-deformación de la Fig. 4. La placa fue modelada como un sólido deformable en 2D y discretizada con 4000 elementos con un control estructurado de la malla. El rodillo fue creado como un alambre rígido discreto con 222 elementos. Debido a la simetría del sistema, sólo se modelo un rodillo y la mitad de la placa.

La Fig. 6 muestra la malla ampliada de una zona del sistema utilizada en la simulación. Debido al tiempo de cálculo excesivo para cada paso de laminación, sólo se simuló una secuencia de cinco pasos, Fig. 7.

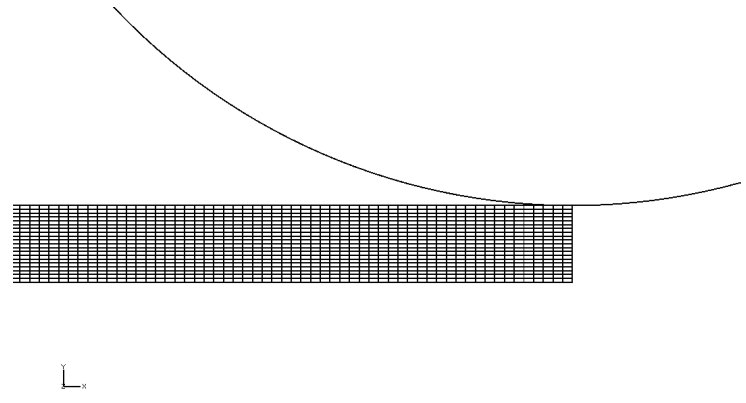

Fig. 6 Discretización del sistema de laminación.

a)

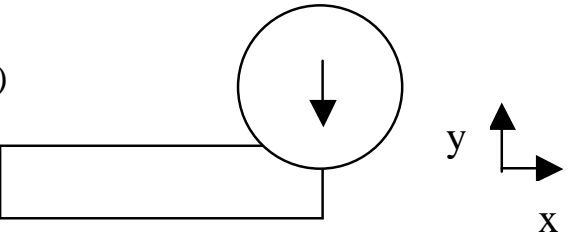

b)

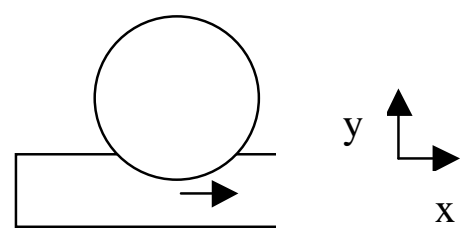

c)

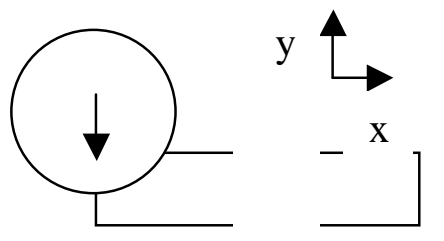

d)

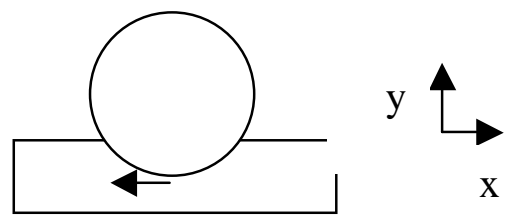

Fig. 7. Secuencia de pasos especificados en Abaqus ${ }^{\circledR}$ para el proceso de laminación.

En Fig. 7: a) el rodillo se desplaza a la posición de la primera pasada y deforma en compresión simple un extremo de la placa, b) El rodillo comienza a girar a la velocidad de laminación hasta que toda la placa sufre la primera pasada de laminación, c) el rodillo se desplaza a la posición de la segunda pasada y deforma en compresión simple el extremo opuesto de la placa, y d) el rodillo comienza a girar a la velocidad de laminación en el sentido contrario a b) hasta que toda la placa sufre la segunda pasada de laminación. Al final de ésta última 
pasada el proceso se repite hasta completar las pasadas requeridas. Los diagramas no están a escala.

\section{RESULTADOS Y DISCUSIÓN}

A partir de los resultados experimentales, del proceso de laminación en frío de las placas de aluminio, se calculó la distribución de fuerza media del rodillo como una función del número de pasos de laminación, Figs. 8 y 9.

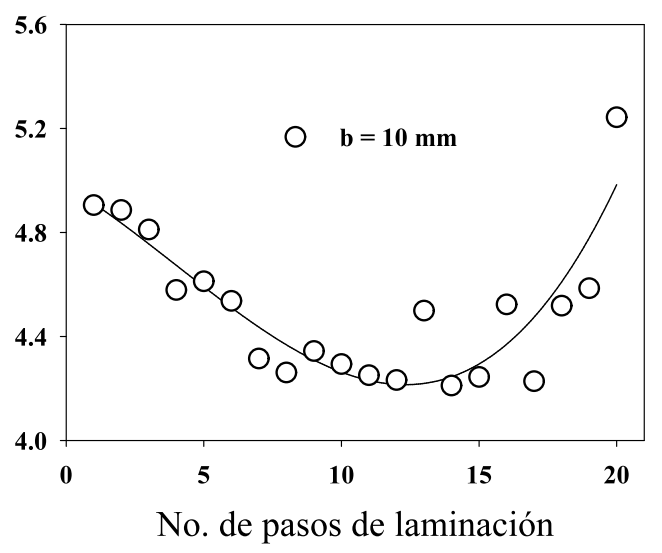

Fig. 8 Fuerza media ejercida por el rodillo en función del número de pasos de laminación para la placa de ancho de $10 \mathrm{~mm}$.

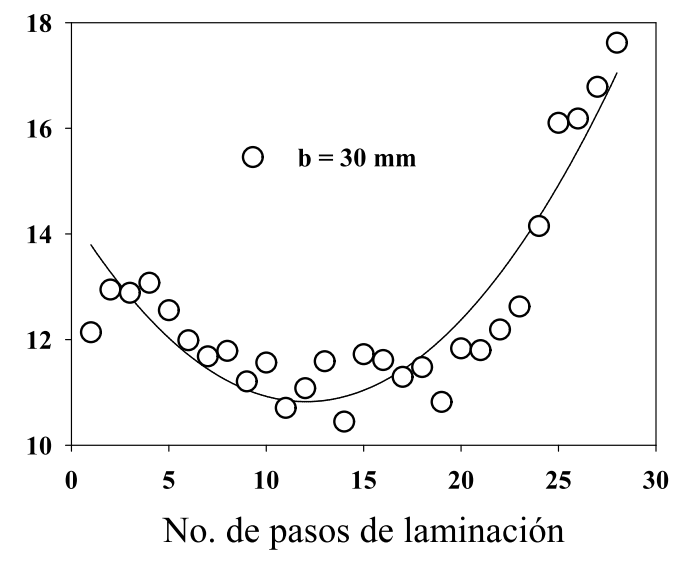

Fig. 9 Fuerza media ejercida por el rodillo en función del número de pasos de laminación para la placa de ancho de $30 \mathrm{~mm}$.

Se observa que la $P_{m}$ del rodillo disminuye al incrementarse el número de pasos de laminación hasta un límite entre los 10 y 15 pasos, rango en el cual se obtiene el $50 \%$ de deformación en espesor aproximadamente. Después del punto $P_{m}$ la fuerza se incrementa paulatinamente hasta alcanzar un valor máximo de $5.2 \mathrm{kN}$ para la placa de $10 \mathrm{~mm}$ de ancho, con $81.56 \%$ de trabajado en frío, mientras que para la placa de $30 \mathrm{~mm}$ un valor máximo de $17.62 \mathrm{kN}$ y $90.34 \%$ de trabajo en frío. Esto se puede explicar cómo que las placas sufren un endurecimiento superficial en los primeros pasos de laminación, lo cual permite que se comporten como materiales elástico-plástico ideales. Después de este punto el material endurece homogéneamente, incrementándose la fuerza media del rodillo. El aluminio se puede aproximar a un metal elástico-plástico ideal para deformaciones plásticas pequeñas, además presenta un coeficiente de endurecimiento bajo o nulo bajo ciertas condiciones de deformación (Khan, 2010).

La geometría en estos casos juega un rol importante en los primeros pasos de laminación, dado que la razón de decremento de $P_{m}$ es proporcional al incremento del ancho de placa. Esto se debe simplemente al ensanchamiento e incremento del área de contacto.

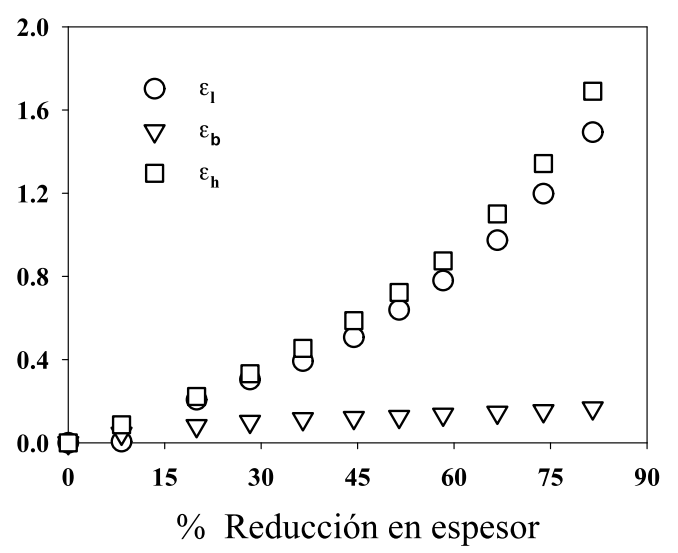

Fig. 10 Efecto del porcentaje de reducción sobre el estado de deformaciones para el ancho de placa de $10 \mathrm{~mm}$.

Las Figs. 10 y 11 presentan el comportamiento de las deformaciones respecto al porcentaje de reducción en espesor, a diferentes anchos de placa. Las deformaciones de la longitud de la placa y de la reducción en el espesor se 
incrementan exponencialmente respecto a la deformación en el ancho, la cual es la menor respecto a las otras. Se debe recordar que lo que se busca es la deformación plana, o sea un ensanchamiento nulo.

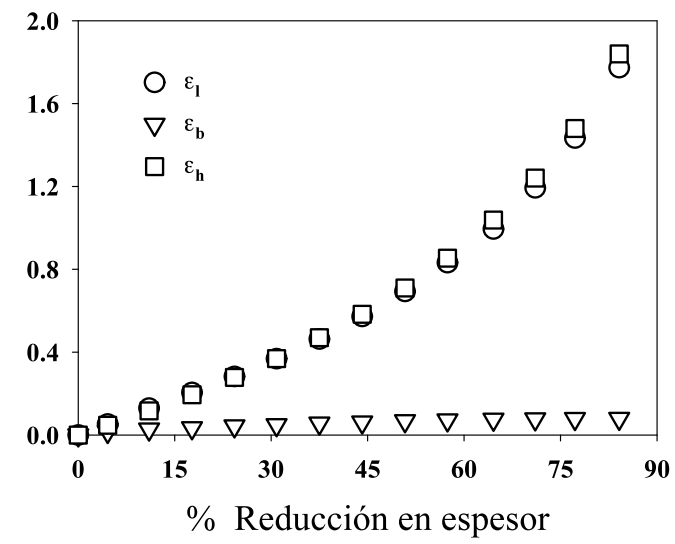

Fig. 11 Efecto del porcentaje de reducción sobre el estado de deformaciones para el ancho de placa de $30 \mathrm{~mm}$.

Por otro lado, las deformaciones longitudinales y en espesor son ligeramente mayores al incrementarse el ancho de placa, de 10 a $30 \mathrm{~mm}$, Fig. 9. Esto se debe simplemente a que al aumentar el ancho de placa las condiciones geométricas de la pieza satisfacen de manera más adecuada la condición de deformación plana, i.e. se considera una placa de dimensión infinita donde las deformaciones en dos de sus dimensiones son mayores en comparación a la otra (ver Fig. 12). De acuerdo a lo anterior, se puede suponer que la condición de deformación plana se cumple para el proceso de laminación plana.

Las Figs. 13 y 14 muestran la evolución del campo de esfuerzos con el criterio de fluencia de von Mises y el campo de deformaciones durante el proceso de laminación después de 1, 2, 3, 4 y 5 pasadas, cuando la placa se encuentra a la mitad del recorrido para cada pasada. Durante la primera pasada se observan dos aspectos de la laminación plana de aluminio:

1) El máximo esfuerzo que se desarrolla es alrededor de los $300 \mathrm{MPa}$ cercano a la superficie, $\mathrm{y}$
2) El estado de esfuerzos decrece en la dirección transversal.

La resistencia a la fluencia de este material se encuentra entre 135-155 MPa por lo que se espera deformación plástica en la superficie y en los alrededores inmediatos, como lo confirma la Fig. 14a.

Esto quiere decir que, al menos, durante la primera pasada el material se deforma heterogéneamente. En la segunda pasada del material entre los rodillos el esfuerzo para continuar deformando se incrementa, así como la deformación plástica.

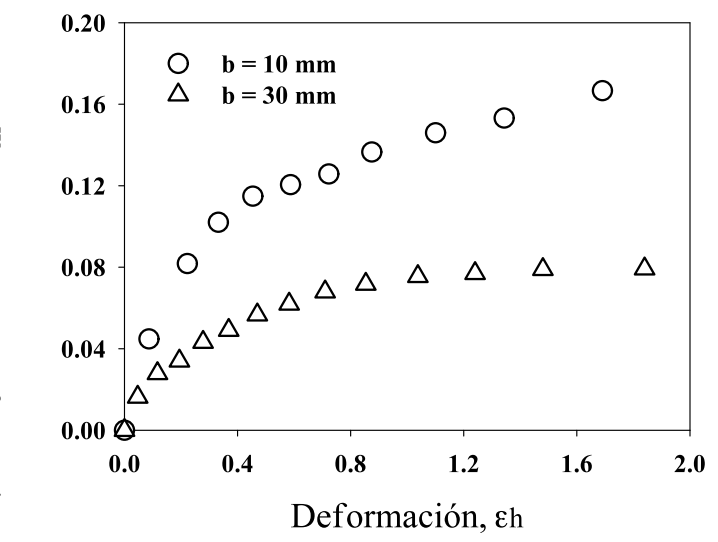

Fig. 12. Efecto de la deformación en espesor sobre la deformación en ancho a 10 y $30 \mathrm{~mm}$.

Se observa en la Fig. 13b que ahora el esfuerzo aumenta alrededor de los $450 \mathrm{MPa}$ y que la deformación, aunque sigue aumentado debido a que el esfuerzo sigue aumentando, con referencia al paso anterior, disminuye (en el primer paso se obtuvo una deformación aproximada de $0.2 \mathrm{~mm} / \mathrm{mm}$ y en el segundo paso de $0.12 \mathrm{~mm} / \mathrm{mm}$, con un acumulativo de 0.32 como se observa en la Fig. 15.

Esto indica que hubo un endurecimiento superficial, lo cual conlleva a que el esfuerzo de fluencia del material aumente, por lo que se requiere de un mayor esfuerzo para continuar deformando. También, a partir de la segunda pasada, el centro de la placa rebasa su resistencia a la fluencia original lo que hace que se presente deformación plástica. 


\section{T. Robert et al.}

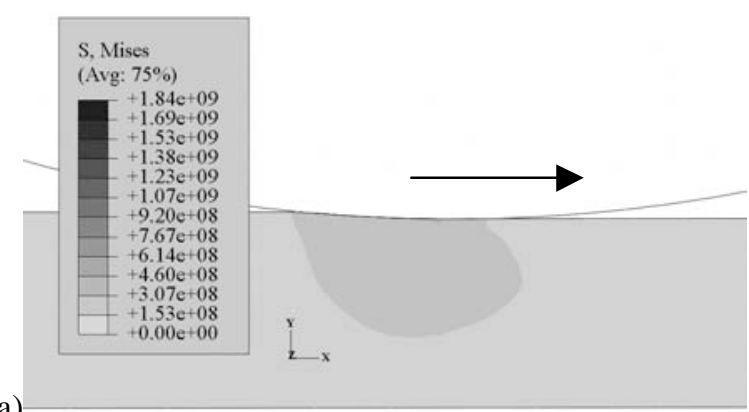

a)

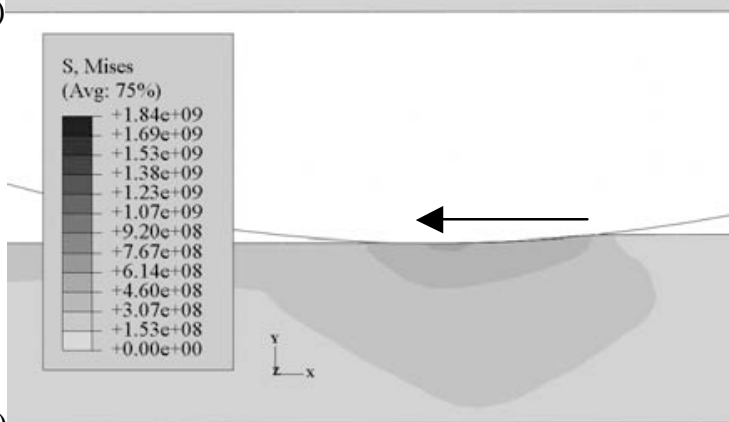

b)

c)

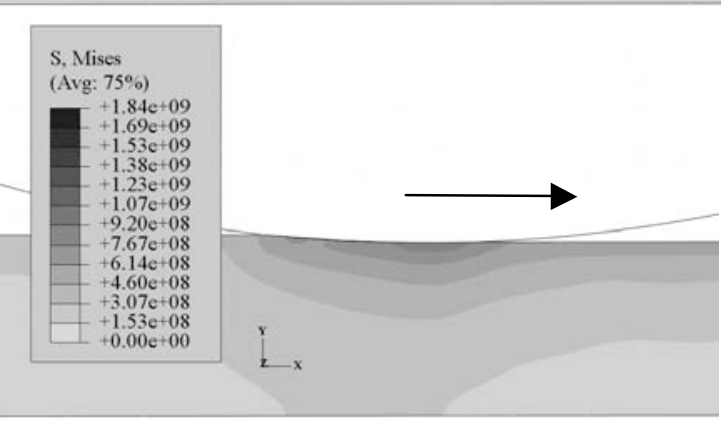

d)
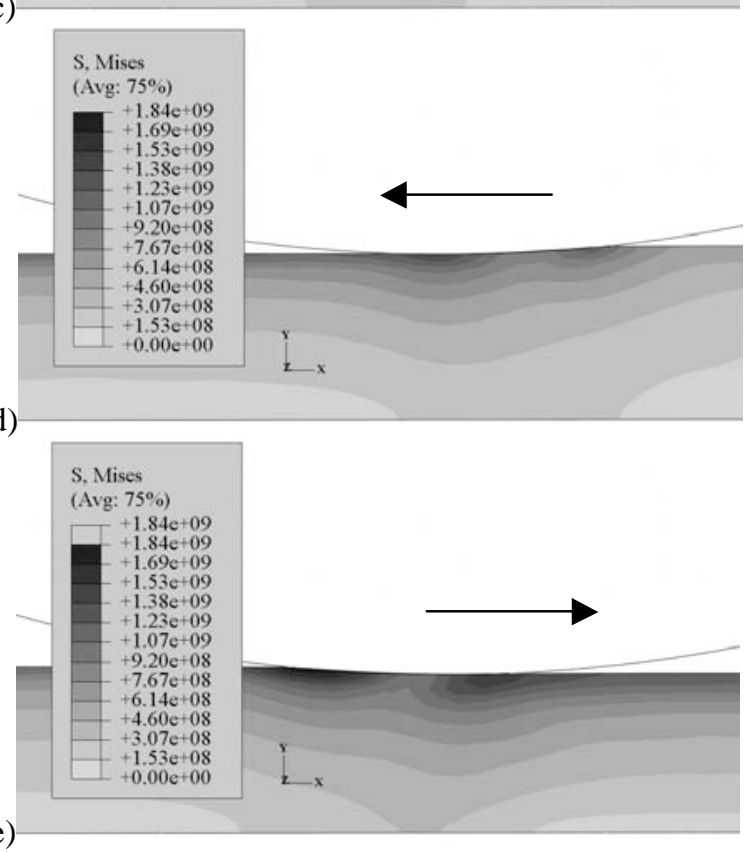

Fig. 13. Campo de esfuerzos desarrollado durante el proceso de laminación después de: a) 1 paso, b) 2 pasos, c) 3 pasos, d) 4 pasos y e) 5 pasos. Las flechas indican la dirección de la laminación.

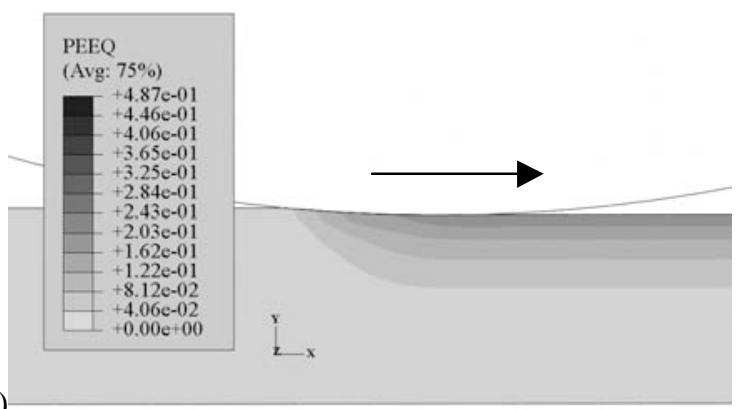

a)

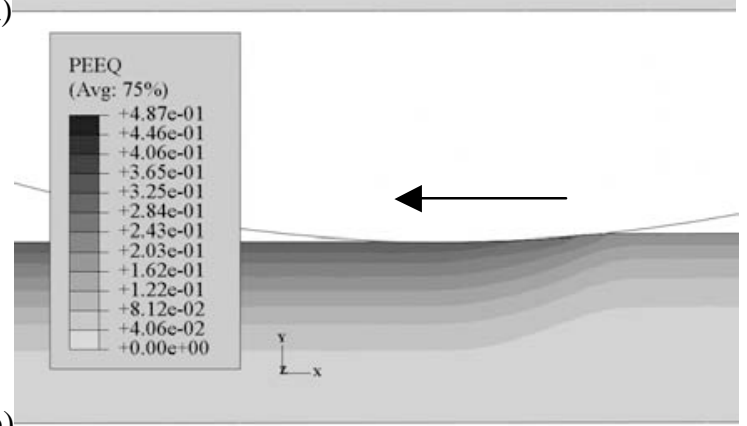

b)

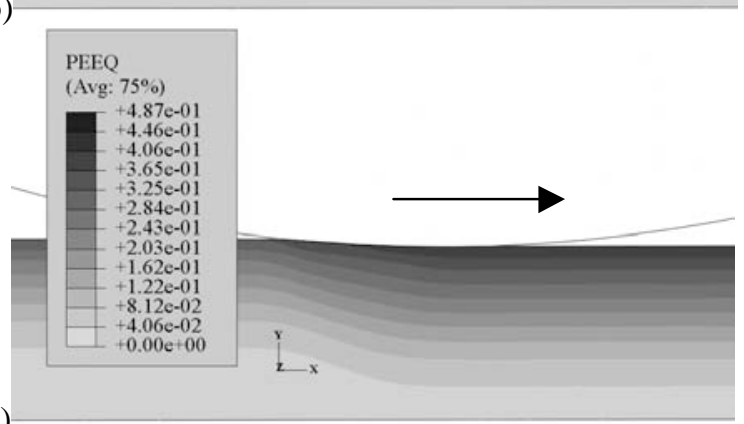

c)

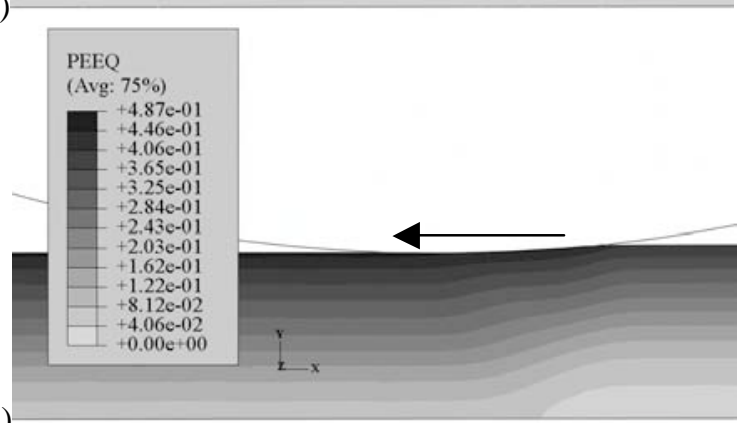

d)

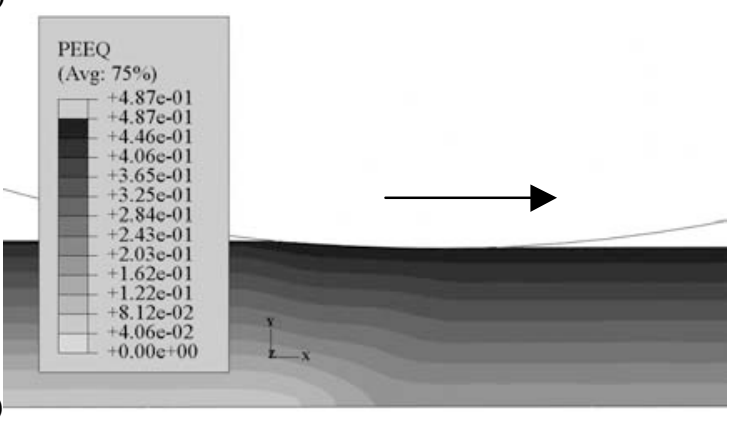

Fig. 14. Campo de deformaciones desarrollado durante el proceso de laminación después de: a) 1 paso, b) 2 pasos, c) 3 pasos, d) 4 pasos y e) 5 pasos. Las flechas indican la dirección de la laminación. 
La Fig. 15 presenta la evolución del campo de deformaciones en la dirección transversal de la parte central de la placa. En las primeras pasadas la deformación plástica se presenta en la superficie y en las cercanías de ésta, conforme el proceso avanza, la superficie se vuelve cada vez más resistente por lo que se necesita un mayor esfuerzo para continuar deformándola; no obstante, el interior poco deformado plásticamente tiene una menor resistencia mecánica por lo que, después de cada paso, la diferencia de deformación permanente se vuelve mayor en el centro de la placa respecto a la deformación en la superficie, Fig. 16. En las pasadas subsiguientes, el estado de esfuerzos en la superficie de la placa aumenta progresivamente debido a que, como se comentó con anterioridad, la superficie sufre endurecimiento por deformación y este endurecimiento va disminuyendo en la dirección transversal hacia el centro de la placa.

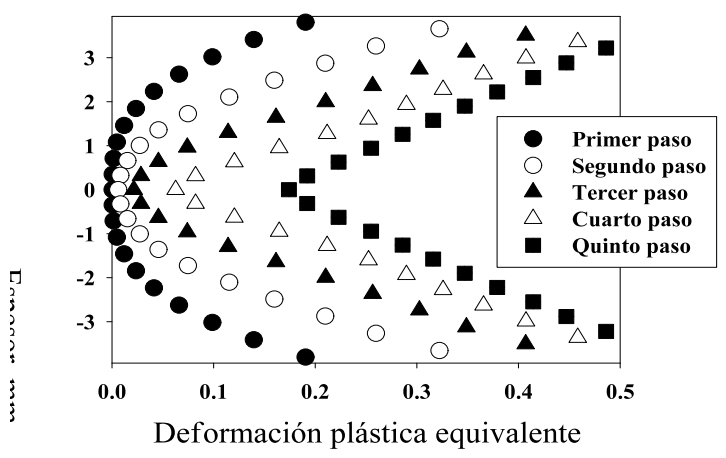

Fig. 15 Campo de deformaciones desarrollado en cada pasada a lo largo de la dirección transversal en la parte central de la placa laminada.

Esto último se presenta porque en las capas centrales del material existe un estado de esfuerzos de magnitud menor, lo que lleva a una menor deformación plástica. También se observa en la Fig. 16 en las pasadas subsiguientes a la segunda que el centro de la placa ahora se deforma más rápidamente que la superficie debido a que como este no se había deformado en los pasos anteriores, sólo se requiere que se llegue al esfuerzo de fluencia original para producir flujo de material.
Se deduce que el comportamiento de la deformación en la superficie es diferente que en el centro, debido a que la superficie cada vez admite menos deformación, lo que hace que la mayor parte de la deformación la soporte el centro de la placa en los pasos subsiguientes.

Al final el material está ya muy endurecido y el espesor es muy pequeño, lo cual incrementa la fuerza para producir una deformación adicional.

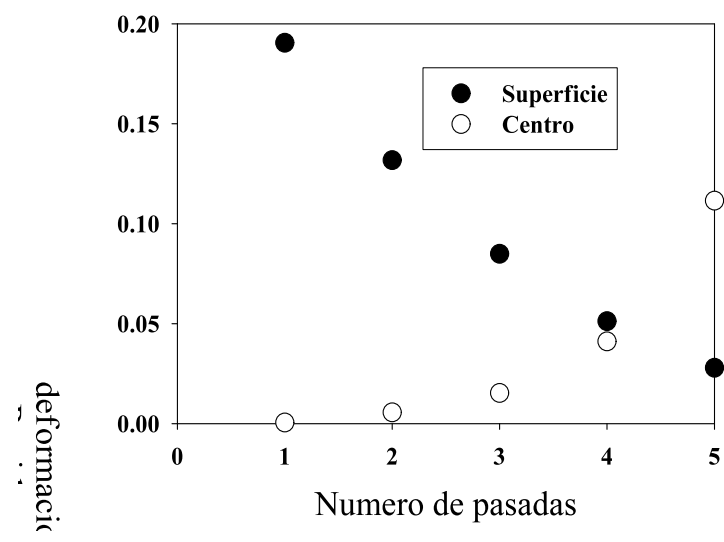

Fig. 16 Rapidez de incremento de deformación plástica en función del número de pasos determinada en la superficie y en el centro de la placa laminada.

\section{CONCLUSIONES}

El proceso de laminación plana en frío del aluminio 6063 involucra un fuerte estado de deformaciones plásticas, obteniéndose un gran trabajado en frío sin sufrir un severo endurecimiento del material.

El incremento del ancho de placa es satisfactorio para el cumplimiento de la condición de deformación plana presentándose mayores incrementos en deformación en la longitud y en el espesor en comparación con el ancho. Por otro lado, se considera un endurecimiento superficial en los primeros pasos de laminación.

La modelación matemática del proceso de laminación permite analizar los estados de esfuerzos y de deformaciones que se generan dentro de las placas de aluminio. Con el modelo matemático se pudo constatar que efectivamente 
existe un proceso de endurecimiento superficial y que por consiguiente el tipo de deformación que prevalece en el proceso de laminación es heterogénea.

\section{NOTACIÓN}

$\begin{array}{llr}h & \text { Espesor } & (\mathrm{mm}) \\ l & \text { Longitud } & (\mathrm{mm}) \\ b & \text { Ancho } & (\mathrm{mm}) \\ v_{r} & \text { Velocidad del rodillo } & (\mathrm{rpm}) \\ R & \text { Radio del rodillo } & (\mathrm{mm}) \\ L_{p} & \text { Longitud de contacto } & (\mathrm{mm}) \\ A_{c} & \text { Área de contacto } & \left(\mathrm{mm}^{2}\right) \\ P & \text { Fuerza laminación } & (\mathrm{kN}) \\ P_{m} & \text { Fuerza laminación } & (\mathrm{kN}) \\ & \text { media } & \\ p & \text { Presión específica } & (\mathrm{MPa}) \\ P r & \text { rodillo } & (\mathrm{MPa}) \\ & \text { Presión radial } & (-) \\ \varepsilon_{h} & \text { Deformación en } & \\ & \text { espesor } & (-) \\ \varepsilon_{l} & \text { Deformación en } & (-) \\ \varepsilon_{w} & \text { longitud } & \text { Deformación en ancho } \\ V & \text { Volumen } & \left(\mathrm{m}^{3}\right) \\ S & \text { Superficie } & \left(\mathrm{m}^{2}\right) \\ n_{i} & \text { Vector unitario } & (-) \\ \sigma_{i j} & \text { Tensor de esfuerzos } & (\mathrm{Pa}) \\ P E E Q & \text { Deformación plástica } & (-) \\ & \text { equivalente } & (\mathrm{Pa}) \\ S . M i s e s & \text { Criterio de fluencia de } & \text { von Mises }\end{array}$

\section{REFERENCIAS}

Chen, W. \& Han, D. (1988). Plasticity for Structural Engineers. EUA: Springer-Verlag.

Dieter, GE (1986). Mechanical Metallurgy. EUA: McGraw-Hill.

Duan, X. \& Sheppard, T. (2002). Three dimensional thermal mechanical coupled simulation during hot rolling of aluminium alloy 3003. International Journal of Mechanical Science, Vol. 44, No. 10, pp. 2155-2172. doi: $\underline{10.1016 / \mathrm{S} 0020-7403(02) 00164-9}$
Khan, M., Hainsworth, S., Fitzpatrick, M. \& Edwards, L. (2010). A combined experimental and finite element approach for determining mechanical properties of aluminium alloys by nanoindentation. Computational Materials Science, Vol. 49, No. 4, pp. 751-760. doi: 10.1016/j.commatsci.2010.06.018

Lozada, O. (2009), Desarrollo de un guión experimental: laminación. Tesis de Licenciatura. Universidad Nacional Autónoma de México. México D.F., México.

Riahifar, R. \& Serajzadeh, S. (2007). Threedimensional model for hot rolling of aluminum alloys. Materials and Design, Vol. 28, No. 8, pp. 2366-2372. doi: 10.1016/j.matdes.2006.08.011

Swank, J. (1892). History of the Manufacturers of Iron in All Ages. Philadelphia, EUA: Allen Lane \& Scott.

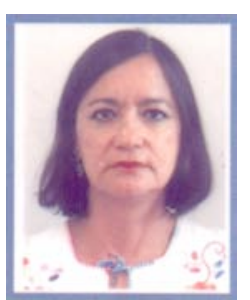

Teresa Robert es Ingeniera Química Metalúrgica, con Maestría en Ingeniería Mecánica, ambas en la Universidad Nacional Autónoma de México. Su área de trabajo es el conformado mecánico de los metales. Actualmente es docente en la Facultad de Química de la UNAM. 